\title{
Cognitive Translation Studies over the Last Decade: Review and Prospect
}

\author{
Shuang Lu \\ Chengdu Jincheng College, Chengdu, China \\ Email: 1020042066@qq.com
}

How to cite this paper: Lu, S. (2021) Cognitive Translation Studies over the Last Decade: Review and Prospect. Open Access Library Journal, 8: e7765.

https://doi.org/10.4236/oalib.1107765

Received: July 16, 2021

Accepted: August 8, 2021

Published: August 11, 2021

Copyright () 2021 by author(s) and Open Access Library Inc.

This work is licensed under the Creative Commons Attribution International License (CC BY 4.0).

http://creativecommons.org/licenses/by/4.0/

\begin{abstract}
This paper reviews and examines the evolution of cognitive translation studies at home and abroad over the last decade. It focuses on three specific theories under cognitive translation studies: Relevance Theory, Adaptation Theory, conceptual metaphor and metonymy, as well as the fundamental trend of cognitive translation transforming from traditional translation study to modern one. This paper, based on the review, aims to explore the current trend in cognitive translation studies, in order to better understand its theoretical framework and application.
\end{abstract}

\section{Subject Areas}

Linguistics

\section{Keywords}

Cognitive Translation, Relevance Theory, Adaptation Theory, Conceptual Metaphor, Conceptual Metonymy

\section{1. 引言}

翻译学是一门研究翻译理论、翻译实践和翻译现象的学科, 具有跨学科、 多学科的性质, 其涵盖语言学、哲学、文化学、文学等多种领域。翻译学借 助语言学建构了很多理论, 仅就语言学理论而言, 翻译学就根据结构主义、 功能主义、转换生成等学派提出了一个又一个新理论。Toury 曾指出 “Theory formation within Translation Studies has never been an end in itself。” ( “翻译 研究中的理论建构永无终结” ) [1]。21 世纪认知语言学的兴起也对翻译研究 产生了重大影响, 促成了一门新兴边缘学科 “认知翻译研究” 的诞生。起始 于 20 世纪 80 年代的翻译过程研究 (translation process research, 简称 TPR)运 用有声思维法(think-aloud protocol, 简称 TAP)、键盘记录法(key-logging)、 
屏幕录制法(screencast)、事件相关电位(event-related potential, 简称 ERP, 俗 称脑电)、眼动跟踪技术 (eye-tracking)、功能磁共振影像(functional magnet resonance imaging, 简称 fMRI)、功能性近红外光谱技术 (functional near-infrared spectroscopy, 简称 fNIRS) 等技术研究翻译行为的认知心理过程 及其神经机制。学界将这种以认知加工过程为导向的翻译研究称为 “翻译认 知研究” (Cognitive Research of Translation) [2]。

谭业升在其《认知翻译学探索: 创造性翻译的认知路径与认知制约》(2012) 一书中指出 “认知翻译学就是有关翻译所涉及的认知过程和译者的认知能力 构成的学科或理论” [3]。需要注意的是, 就像翻译作为一个学科在创立初期 之时, 也只是被称作 “翻译理论” 或者 “翻译研究”, 而不是 “翻译学” 一 样, “认知翻译” 尚未形成学科体系, 因此笔者在此保守地采用 “认知翻译 研究” 这一提法。2010 年出版的《翻译与认知》一书就是在这种学术背景下 诞生的一部开拓性著作。Shreve \& Angelon 认为, 翻译的未来方向为 “从认 知角度研究翻译”, 且在近期将会硕果累累; Halverson 随后指出, 我们必须 明确地沿着认知理论向前发展翻译学 [4]。Martin 也持相同观点, 且在前人研 究的基础上, 直接提出了 “认知翻译学” ( “Cognitive Translatology”) 这一术 语, 阐述了认知翻译学的研究范式, 以及认知翻译学的相关学科问题, 标志 着该术语在翻译学领域中地位的确立, 主张在该学科中尽快建立理论与实践 之间的互动研究 [2]。认知翻译学把译者及其翻译过程确定为研究的主要对 象, 主要研究译者的认知风格、智力水平、翻译能力、思维特点、语言认知 机制等。“译者研究的广义原则大纲将涵盖社会学、文化和认知, 以不同于 以往的方式来看待译者的能动作用或使然过程。”认知翻译学家认为可以用 科学方法和先进仪器探索译者心理活动的 “黑箱子” (black box), 比如, 可以 运用语料库语言学的方法来研究翻译过程及其翻译作品, 通过检索翻译文本 的语料库从而对译作质量和特点进行详尽的描述。除此之外, 认知翻译研究 密切关注翻译过程的技术化。近年来, 机器翻译的日益普及使得认知翻译研 究学者重点分析译者在翻译过程中管理和控制译出语言的过程及其方式, 通 过仔细观察译者在翻译过程中的思路以及如何解决在翻译时遇到问题等, 从 而探讨译者能力对译作质量的影响。另外, 认知翻译研究借用心理学和语言 测试学的手段来分析和评估译者的能力, 把韦氏成人智力量表(Wechsler Adult Intelligence Scale, 简称 WAIS) 用于评估和评价译者的智力结构和智力 特点, 用 TOEFL 的分测验来评价译者的语言认知特点或语言背景知识 [2]。

基于国内外认知翻译研究近十年的发展历程, 本文将对认知翻译研究下 的具体三大理论: 关联理论、顺应理论、概念隐喻和转喻的认知翻译的研究 进展进行探究, 旨在探讨当前认知翻译研究的趋向, 以期更好地充实其理论 架构及其应用。

\section{2. 近十年认知翻译研究的进展}

严格说来, 认知翻译研究最早始于对语用学中有关关联理论以及顺应论 的探讨与研究。后来随着认知科学尤其是认知语言学的发展, 翻译被重新定 义为 “既是一门科学, 也是一门艺术, 是科学与艺术的统一”。 


\section{认知翻译研究理论的探讨}

Martin 首创了 “认知翻译学” 这一术语, 王寅于 2005 年在《中国翻译》 上根据 Lakoff、Johnson、Langacker 等所倡导的 GL 基本原理最初论述了 “认 知翻译观”, 初步阐述了从 GL 角度建构翻译理论的思路 [5]。此外, 他在《认 知语言学的 “体验性概念化” 对翻译中主客观性的解释力一一一项基于古诗< 枫桥夜泊> 40 篇英语译文的研究》一文中还运用 GL 中的 “体验观” 和 “识 解观” 分析了同一首唐诗 “枫桥夜泊” 的 40 篇译文。他发现这些译文之所以 存在差异, 是因为这 40 位译者各自有不同的认知方式, 因而择用了不同词句, 这体现了人文主观精神 [6]。后来, 受到 Martin 提出的认知翻译学的启发, 王 寅运用体验哲学和 CL 的基本观点论述了认知翻译研究的具体原则及方法。 他首先梳理了国内外翻译界在翻译过程研究中借用的认知科学的若干研究方 法以及研究成果, 包括记忆结构(Memory Structure)、元认知(Metacognition)、 眼动跟踪实验(Eye Tracking)、功能性磁共振成像(fMRI)等。此外, 他大力倡 导的语言的 “体认观” , 试图将 CL 的核心原则归结为 “现实 - 认知 - 语言” 三个过程, 也就是把语言的形成归结于对现实世界进行互动体验和认知加工 [7]。随着研究的深入, 王寅把认知语言学和认知翻译学研究上升到后现代哲 学层面进行进一步探讨, 通过叙述欧洲学者们提出的十数种实证方法、研究 工具及其适用范围和评价其优缺点后, 他大力倡导认知翻译研究的重中之重 是将理论与实验紧密结合, 并融入各种语料库和实验方法来证明提出的相关 论点及种种假设, 以便更好地在后现代哲学视野下建构和实践认知翻译学。 也就是说, 认知翻译研究首先应立足于翻译学或语言学阵地, 从哲学等高度 建构理论系统; 同时运用先进设备做实验, 用数据说话 [8]。王寅基于认知过 程的感觉、知觉、意象、意象图式、范畴、概念、意义、(理想化)认知模型、 ECM、隐转喻十个环节, 尝试以其为基础来具体论述翻译过程的认知操作和 运行步骤, 以期能在认知翻译学的理论框架中深入探索翻译过程所涉及的具 体因素。但同时他也认识到局限性：语言 “认知过程” 十分复杂, 所能罗列 进行说明的也不过只是一个大致分析; 翻译过程”本身也是一个复杂的机制, 也难以一概而论。该文章最终揭示了在整个认知过程或翻译过程中, 每一个 节点都可能出现多种选择的可能性, 因此对同样的作品就可能出现不同人的 多种译法, 甚至同一译者因自身境遇和情绪也会有不同的译法[9]。文旭则首 先肯定了近年来随着认知科学尤其是认知语言学的发展, 将认知语言学与翻 译学的研究结合起来已成为新的一大趋势。他分别从认知语言学的哲学观、 语言观和表征方法视角出发, 探讨了认知翻译学的一些问题。同时他也提到 就翻译标准这一问题, 应是 “仁者见仁, 智者见智”, 这一点与王寅的观点 不谋而合。最后, 他总结到: 翻译就是一种认知过程, 是不同语言或语言变 体之间的范畴转换, 这种转换涉及人类认知的一些基本特征 [10]。随着人工智 能技术的发展, 谭业升指出: 在新的经济全球化历史时期, 在政治、文化政 策和翻译技术的推动下, 翻译职业的重新组织和定位将更加侧重跨文化干扰 等因素形成的人类翻译附加值 [11]。

中国认知翻译研究领域的首部专著《跨越语言的识解一一翻译的认知语 言学探索》于 2009 年诞生, 作者为谭业升。它吸收了关联翻译理论和翻译图 
式研究的成果, 结合认知语言学的基本理论假设, 创建了翻译中意义建构的 认知模式, 最后主张翻译学应在认知科学中占有一席之地 [12]。此外, 他的另 一部专著《认知翻译学探索: 创造性翻译的认知路径与认知制约》于 2012 年 出版, 是上一部专著的续篇, 是从认知科学的一个重要分支即认知语言学的 角度对翻译现象进行探究[7]。

\section{3. 具体认知翻译学理论的探讨}

随着认知语言学的深入发展, 国内外学者也顺应国际认知翻译学研究的 潮流与趋势, 逐步将认知语言学与翻译结合起来进行研究与探讨。如今, 已 经在关联理论、顺应论、概念隐喻和转喻的认知翻译等多个领域取得了一定 进展。

\section{1. 关联理论的探讨与研究}

关联理论的翻译观是以 20 世纪 80 年代兴起的关联理论为基础的。关联 理论认为, 语言交际是一个涉及信息意图和交际意图的 “明示一一推理” 过 程。说话人提供最大的关联信息，听话人则根据语境或认知环境对信息进行 推理，选择关联性最大的假设，获取最佳关联，从而达到交际目的。恩斯特 - 奥 古斯特 - 格特(Earnst-August Gutt)最先根据关联理论对翻译进行研究, 在《翻 译与关联: 认知与语境》一书中巧妙地将语用学中的关联原则与认知心理学 普遍原理结合起来, 提出了关联翻译论(relevance translation theory)[13]。林 克难首次把关联翻译理论引进了我国翻译界，提出翻译是两种语言之间特殊 形式的交际, 翻译的过程本质上是也译者的推理过程[14]。综观国内关联翻译 研究这十多年来的发展历程, 关联翻译理论主要被应用在诗歌翻译中。

谈到诗歌翻译, 究竟是否存在诗歌翻译的不可译性, 关联理论认为, 说 话人可以将自己的意旨传达给另一方，不仅可以将意思而且可以将内容和方 式同时传达出来。翻译毕竟是一种言语交际活动, 而交际成功取决于一方的 意图被另一方识别[15]。按照这一观点来看, 不存在不可译性。因此, 段鼻卉 在关联理论的框架下对诗词翻译这一交际过程进行研究, 并对唐代诗人杜甫 的《春望》原文进行关联理论分析, 通过译文对比来探析译者翻译时的认知 推理过程。通过对比发现：诗词文本意义本身的高度不确定性导致不同译者 对同一事物的认知出现差异，于是造成同一诗出现多种译本 [16]。关联理论对 诗歌翻译有着很强的解释力。“译者首要的任务是达到翻译的效度使原文作 者的意图与译文接受者的期待相吻合, 其次有责任提高译文的信度, 使译语 文本最大程度地向原语文本趋同。”[15]

莫丽红, 戈玲玲从关联翻译理论的视角研究汉语成语的翻译, 将汉语成 语的翻译视作是一种 “认知一一推理” 的交际过程。并提出在此过程中, 译 者应综合考虑原文作者的意图和译文读者的认知环境等因素，灵活采用不同 的翻译策略, 准确地向译文读者传达原文作者的意图[17]。因此，对翻译过程 进行认知解释性研究仍将是关联翻译论发展的主流取向。关联理论在一定程 度上消解了传统认知研究所固有的思辨色彩, 从而使翻译研究更加科学化。 


\section{2. 顺应理论在翻译实践中的应用}

Jef Verschueren 提出顺应理论, 并认为语言使用是一种社会行为, 应从 认知、社会、文化的角度综合分析其语言行为[18]。而翻译作为多种语言间的 交际活动, 就是译者为顺应各种语境而不断做出选择的过程。因此翻译能力 应为译者作选择时所拥有的潜在的待选项的总和, 翻译行为则指实际翻译即 选择的结果。将顺应论应用于翻译实践中, 译者能对翻译策略的选择有一个 全新的视角。但以 Verschueren 的顺应论视角研究翻译, 在国外的相关研究 中尚未看到。目前在国内, 顺应论运用的最为广泛的领域要数翻译。

宋志平, 徐珺从译者的角度出发, 认为应该对语言学习错误和翻译选择 错误二者进行明确区分。他们认为前者属于二元对立错误, 而后者则呈非二 元对立性。因为二元性错误为语言能力不足所致, 例如: 例如不少人将 “ We still call the copper-colored natives of the New World Indians” 译成 “我们仍把 古铜色皮肤的土著称作新大陆印第安人”。事实上, 此为误译。但误译的原 因不在于译者对翻译技巧运用不当, 而是语法意识不足所致。也就是说, 译 者没有真正理解这句话的意思: “我们仍把新大陆古铜色皮肤的土著称作印 第安人。”而翻译活动的前提是译者已具备足够的双语能力, 因而翻译错误 应当属于非二元对立错误。例如, 把 “There are no winners in a divorce” 译 成 “在离婚中没有胜者” 还是 “离婚的人都是两败俱伤” , 选择任何其一的 表达都必然有其道理, 如果没有特定的上下文, 就不存在正误问题。除非在 极特殊的情况下, 否则没有所谓好译文和坏译文之分。也就是说, 真正发生 在翻译过程中的所谓错误则关涉译者的选择问题, 而这些选择受制约于译者 意识到或未意识到的多重因素, 如特定的语境、对接受者的考虑以及译者本 人的文化观、翻译观等呈现出非二元对立性[19]。

李占喜参照顺应论的理论框架提出文学翻译的顺应论, 从语境关系顺应、 语言结构顺应(语音层面、词语层面、句子层面和超语句层面顺应的动态性) 以及翻译过程中译者的语用意识等相互联系的方面, 从一个新的角度对翻译 过程进行阐释。他将译者在翻译过程中对目的语文本做出恰当选择的原因归 纳为目的语与源语具有三个同样的特征, 即变异性、商讨性和顺应性。作者 在文中提到, 在翻译过程中, 除了传达字面意思之外, 更应该兼顾到源语语 言的音韵美感的传递, 这样会使译文更好地传达出原文作者的意图。例如, 作者举例在翻译 “Home is home, but it ever so homely” 这一句话时, 除了将 英文原文的字面意思以及语言风格传达到位之外，还要考虑传达 “Home”,

“home” , “homely” 三个词的音韵, 为顺应中国文化的认知语境, 作者最 后将之译为 “家虽不佳仍是家” [20]。

翻译中的语境是在翻译过程中译者使用目的语语言阐释源语文化的过程 中动态生成的语境会随着翻译过程的发展而变化。在顺应的过程中, 译者必 须使自己的翻译行为及思维运作顺应于源发语与目的语双方的认知环境, 只 有这样, 他的译文选择才能确保不同文化之间交际活动得以顺利进行。

\section{3. 概念隐喻和转喻的认知翻译}

1980 年, Johnson 和 Lakoff 推出专著《我们赖以生存的隐喻》从认知角 
度全面、系统地对隐喻的本质进行了阐发, 并提出了概念隐喻[21]。他们第一 次站在认知的角度从功能和结构两个方面对认知隐喻进行阐释。由此看来, 隐喻不仅仅是一种修辞手法或者语言现象, 更重要的是一种认知现象, 对人 类思维方式、语言的理解以及许多技术的运用有重要价值。刘冰泉、张否受 到上世纪八十年代出版的《我们赖以生存的隐喻》一书的启发, 区别了隐喻 作为修辞手法和认知方式的理论, 其次从认知这一新视角分析了大量隐喻的 英汉互译的句子和习语, 最后总结了隐喻翻译的策略和方法, 即当源语隐喻 中的源域和目标域能够在目的语中找到对应的源域和目标域时, 可采用映射 对等翻译; 若是无法找到或是只有相类似的源域和目标域, 则采用偏移等效 翻译方法[22]。何立芳、李丝贝则上升到语言背后的跨文化高度, 提到 “中国 英语” 这一概念, 即为使源语和目的语取得文化认知上最大程度的对等, 最 好的办法是在音译或直译的基础上加注释, 翻译界常把这样的译文归为中国 英语(China English 或 Sino-English), 且常被用于翻译承载中华民族深厚文化 底蕴的典籍作品。通过对道教典籍翻译中概念术语的中国英语现象进行探讨, 他们认为在道教典籍翻译中应大胆采用 “中国英语” 传播原汁原味的中国文 化, 为世界英语注入中国元素, 以此展示中国文化的软实力[23]。王天翼、王 寅则认为 “概念隐喻” 作为人类认知层面的一种机制, 不仅在形成概念、思 维、语言的过程中起着举足轻重的作用, 在翻译过程中也十分关键, 通过论 述 “变异” 和 “损耗” 这两条支隐喻机制, 从而揭示了相关隐喻性表达式背 后所隐藏的隐喻机制 $[24]$ 。

谭业升在《转喻的图式一一例示与翻译的认知路径》一书中构建了认知 翻译模式, 从认知科学尤其是认知语言学框架下探讨了在翻译过程中以语境 为基础并受规约限制的转喻图式的例示, 阐释了基于多样性邻接关系的转喻 图式 - 例示级阶与翻译转换、翻译变体的关联, 并提出对今后开展转喻与翻 译关系研究的建议 [25]。张辉, 卢卫中在《认知转喻》一书中则系统性论述了 当代认知语言学中认知转喻理论的研究现状。书中就转喻与语用、语篇分析 以及文学和翻译等方面进行了深入细致的分析和探讨, 尤其是转喻翻译的原 则和策略, 转喻在词典翻译中的应用以及语境对词典转喻翻译的制约 [26]。文 旭、肖开容在《认知翻译学》一书中指出: 认知翻译学目前急需建构恰当的 理论模型, 对翻译中的语言转换和认知机制做出理论分析, 从而形成可供认 知翻译实证研究检验的理论假设 [27]。胡壮麟在《认知隐喻学》一书中基于中 外的比较研究, 着重探讨了隐喻、语言和认知三者的关系[28]。

\section{4. 认知翻译研究未来的发展趋势}

基于以上分析和综述, 笔者发现, 尽管认知翻译研究目前已经解决了一 些其它翻译理论无法解决的翻译问题, 但目前这一研究正处于发展阶段, 许 多理论尚未成熟。因此, 整个研究体系还存在一定的不足和缺陷, 应引起关 注和重视: 关联理论对 “关联性” 界限定义模糊, 会导致译者在翻译中做出 主观性较强的 “关联” 判断, 这是关联理论目前应用到翻译研究中的最大障 碍, 因而关联翻译研究在方法论上目前还有待完善。

纵观国内外认知翻译研究的发展, 笔者认为其未来主要有以下几大趋势: 
首先, 翻译学本身就具有跨学科性, 而认知过程本身也是一种复杂的机 制。越来越多的学者大胆吸收其他学科领域的长处, 让翻译研究的深度和广 度都有所突破, 这是翻译研究的必然趋势。从认知翻译研究的角度出发, 顺 应论将翻译理论纳入一个统一连贯的框架, 为拓宽翻译研究带来了诸多启示。 纽马克指出 “隐喻翻译是一切语言翻译的缩影, 因为隐喻翻译给译者呈现出 多种选择方式: 要么传递其意义, 要么重塑其形象, 要么对其进行修改, 要 么对其意义和形象进行完美的结合, 而这一切又与语境因素、文化因素如此 密不可分, 与隐喻重要性的联系就更不用说了。” [29]这很好地说明了研究隐 喻翻译的重要性, 也概括了隐喻翻译的复杂性。此外, 从认知的角度来看翻 译的话, 不妨将翻译描述为 “将源语言认知方式在目标语中体现” 。尤其是 文学翻译方面, 翻译不单单是简单语言活动, 更上升到了语言背后的跨文化 层面。世界上不同的文化背景产生了不同的思维状态和方式。那么, 译者在 翻译过程中如何超越语言表达的限制从而达到认知意识的默契与协调, 需要 我们借助认知翻译研究的发展不断进行认知推理过程分析才能得出这个答 案。

其次, 中国认知翻译研究应秉承自身特色, 即根据汉语语言本身的特点, 从汉语的实际运用出发, 形成自己的理论和流派。将翻译说成模仿, 意味着 译者既要追求相似性, 也要有所变异, 丢失原作部分信息在所难免。不同民 族之间的政治、历史、文化、习俗、经济和科技的发展存在差异, 因此民族 间也必然存在思维的差异。值得一提的是, 文化负载词的翻译则代表了翻译 界长期关注的 “不可译” 问题, 例如 “仁、义、礼、智、信” “君子” “秀 才” “举人” “进士” 等。尤其是在中国典籍翻译过程中, 为了让源语和目 标语达到文化认知上最大程度的对等, 要做到一方面保留源语喻义, 另一方 面又不舍弃其喻体是译者面临的很大难题。笔者发现, 自 1994 年关联翻译论 引入我国以来, 翻译界对翻译关注的重心从研究静态的文本解码与编码转移 到对译者主体性的探讨, 研究作为交际者的译者的大脑理解和推理机制, 尝 试对 “翻译是如何发生的” 做出科学解释, 发掘出双语转换的思维规律, 从 而为翻译科学的建构打下基础。由此看来, 在认知翻译研究下这一大背景下, 译者主体性再次得到关注和彰显, 因而笔者认为, 译者在翻译过程中无需过 多纠结翻译策略的选择, 采用集中国特色的 “中国英语” 来翻译承载中华民 族深厚文化底蕴的典籍作品, 不失为一种很好的手段。

\section{5. 结语}

基于对国内外认知翻译研究的简单回顾, 可以看出近年来认知翻译研究 取得了一些成果。但考虑到 “认知翻译” 还处于萌芽阶段, 并未形成一个完 整的学科体系, 因此笔者只看到 “认知翻译理论”, “认知翻译观” 或 “认 知翻译研究”, 而非 “认知翻译学” 的说法。通过这一点, 也可以推断出认 知翻译研究只是起到了一种基本的引介作用, 距离建构成学科体系还有很长 一段距离。需引起关注的是, 由于客观上来看, 过于注重强调语言学概念的 嫁接, 如探讨关联理论及顺应理论所占比重较大, 导致在对具体翻译问题进 行分析时, 理论与实际问题尚未实现有机融合。另外一方面需要注意的是, 
同翻译的 “文化转向” 类似, 翻译的 “认知转向” 作为翻译研究的新思路, 也并不仅仅是对翻译所涉及的认知心理过程予以关注, 更是要对前人研究中 帮助我们认识翻译和进行翻译分析的范畴进行重新评估, 因为认知翻译研究 过程中肯定会不可能避免地涉及到对实证翻译研究以及社会环境因素的考 量, 如果仅仅是在有限的认知科学范式框架内对翻译过程进行描述, 那么翻 译的动态性和复杂性将会被大大忽视。只有采用认知科学和人文科学相结合 的路径，才能对翻译这一复杂的活动进行探讨。

翻译活动是一个及其复杂的、包含多种因素的动态过程, 要想进一步获 得有关译者认知过程的更加全面、系统的认识, 还需要借鉴诸多其他学科的 知识, 例如哲学、语言学、心理学、传播学以及神经科学等领域的研究, 当 涉及到译者在翻译过程中的心理状态和神经系统运作的过程, 也需要依托认 知科学其他分支学科的实证研究进行佐证。因此不管是从方法论还是具体实 证研究上都值得进一步探索。希望有朝一日认知翻译研究不管在认知科学领 域, 翻译学领域还是二者有机结合而产生的新兴学科发展中都能占有一席之 地。未来, 认知翻译研究从单一学科研究走向综合式交叉研究大学科是必然 的趋势。

\section{Conflicts of Interest}

The author declares no conflicts of interest regarding the publication of this paper.

\section{References}

[1] Toury, G.A. (1988) Handful of Paragraphs on "Translation" and "Norms". In: Schäffner, C., Ed., Translation and Norms, Multilingual Matters, Clevedon, 10-32. https://doi.org/10.1080/13520529809615501

[2] Martin, R.M. (2009) The Way They Were: Subject Profiling in Translation Process Research. In: Mees, I.M., Alves, F. and Gopferich, S., Eds., Methodology, Technology and Innovation in Translation Process Research: Copenhagen Studies in Language, Samfundslitteratur, Frederiksberg, 87-108.

[3] 谭业升. 认知翻译学探索-一创造性翻译的认知路径与认知制约 $[\mathrm{M}]$. 上海: 上 海外语教育出版社, 2012.

[4] Halverson, S.L. (2010) Cognitive Translation Studies: Developments in Theory and Method. In: Shreve, G.M. and Angelone, E., Eds., Translation and Cognition, John Benjamins, Amsterdam, 349-369. https://doi.org/10.1075/ata.xv.18hal

[5] 王寅. 认知语言学的翻译观 [J]. 中国翻译, 2005, 26(5): 15-20.

[6] 王寅. 认知语言学的 “体验性概念化”对翻译中主客观性的解释力一一一项基于 古诗《枫桥夜泊》 40 篇英语译文的研究[J]. 外语教学与研究, 2008(3): 211-217.

[7] 王寅. 认知翻译研究[J]. 中国翻译, 2012, 33(4): 17-23.

[8] 王寅. 认知翻译研究: 理论与方法[J]. 外语与外语教学, 2014(2): 1-8.

[9] 王寅. 基于认知语言学的翻译过程新观 [J]. 中国翻译, 2017, 38(6): 5-10.

[10] 文旭. 认知翻译学: 翻译研究的新范式[J]. 英语研究, 2018(2): 103-113.

[11] 谭业升. 认知翻译学对翻译研究的重新定位[J]. 中国外语, 2021(3): 79-87.

[12] 谭业升. 跨越语言的识解: 翻译的认知语言学探索 $[\mathrm{M}]$. 上海: 上海外语教育出 
版社, 2009.

[13] Gutt, E. (1991) Translation and Relevance: Cognition and Context. Basil Blackwell, London.

[14] 林克难. 关联翻译理论简介[J]. 中国翻译, 1994(4): 6-9.

[15] 赵彦春. 关联理论对翻译的解释力[J]. 现代外语, 1999(3): 276-295.

[16] 段㮂卉. 关联理论视角下汉诗英译的认知推理过程探析一一以唐诗《春望》五种 译文为例[J]. 外语教学, 2010(4): 96-99.

[17] 莫丽红, 戈玲玲. 关联翻译理论视角下的汉语成语翻译 [J]. 湖南社会科学, 2012(2): 189-194.

[18] Verschuren, J. (2000) Understanding Pragmatics. Foreign Language Teaching and Research Press, Beijing.

[19] 宋志平, 徐珺. 选择顺应论视角下的翻译错误非二元对立性分析[J]. 外语研究, 2009(6): 74-78.

[20] 李占喜. 顺应论: 文学翻译的一个新视角[J]. 华南农业大学学报(社会科学版), 2009, 8(3): 89-97.

[21] Lakoff, G. and Johnson, M. (1980) Metaphors We Live By. The University of Chicago Press, Chicago.

[22] 刘冰泉, 张磊. 英汉互译中的认知隐喻翻译[J]. 中国翻译, 2009(4): 71-75.

[23] 何立芳, 李丝贝. 道教典籍语言隐喻认知特征解析与翻译[J]. 外语学刊, 2017(4): 99-103.

[24] 王天翼, 王寅. 翻译隐喻观的认知分析一一以“变异”和“损耗”两条支隐喻机制为 例 [J]. 外语研究, 2018, 35(3): 82-86.

[25] 谭业升. 转喻的图式一一例示与翻译的认知路径[J]. 外语教学与研究, 2010(6): 465-471.

[26] 张辉, 卢卫中. 认知转喻 $[\mathrm{M}]$. 上海: 上海外语教育出版社, 2010 .

[27] 文旭, 肖开容. 认知翻译学 $[\mathrm{M}]$. 北京: 北京大学出版社, 2019.

[28] 胡壮麟. 认知隐喻学 [M]. 北京: 北京大学出版社, 2020.

[29] Newmark, P. (2001) Approaches to Translation. Shanghai Foreign Language Education Press, Shanghai.

\section{Appendix (Abstract and Keywords in Chinese)}

\section{近十年认知翻译研究：回顾与展望}

摘要: 本文回顾国内外认知翻译研究近十年的发展历程, 阐述近十年来认知 翻译理论发展的相关状况, 着重探讨了认知翻译研究下的三大具体理论: 关 联理论、顺应理论、概念隐喻和转喻的认知翻译所取得的主要进展, 以及翻 译研究从传统走向现代这一基本态势。同时, 基于综述, 本文旨在探讨当前 认知翻译研究的趋向, 以期更好地充实其理论架构及其应用。 关键词: 认知翻译, 关联理论, 顺应理论, 概念隐喻, 概念转喻 